\title{
Hemophagocytic syndrome in a patient with disseminated tuberculosis: a case report
}

\author{
Vania Lombardo, ${ }_{1}^{1}$ Fania Puccia, ${ }^{1}$ Antonino Terranova, ${ }^{1}$ Andrea Affronti, ${ }^{1}$ Ada Maria Florena, ${ }^{2}$ Lydia Giannitrapani, ${ }^{1}$ \\ Maurizio Soresi ${ }^{1}$
}

${ }^{1}$ Biomedical Department of Internal Medicine and Specialities, University-Hospital of Palermo; ${ }^{2}$ Department of Sciences for Health Promotion, Section of Pathology, University-Hospital of Palermo, Italy

\begin{abstract}
Hemophagocytic syndrome (HS) is a rare disorder of the immune system. It is characterized by fever, lymphadenopathy, hepatosplenomegaly, cytopenia and hyperferritinemia. The cause differs in each country suggesting a specific genetic background and epidemiology of infections, and it can be associated with malignant diseases. A rare cause of HS is tuberculosis (TB), we describe a case of HS associated with disseminated Mycobacterium tuberculosis (MT) infection in a patient from Sudan. He presented diarrhea, fever, pancytopenia, thickened and dilated bowel loops and lymph nodes enlargement at ultrasound and computed tomography scan. A bone marrow biopsy performed to rule out a lymphoma revealed a HS. The bronchoalveolar lavage (BAL) culture was then positive for MT and subsequently, radiologic aspects of lung and spleen TB involvement appeared. A disseminated tuberculosis was diagnosed. Despite antituberculous therapy, the patient died as in approximately $50 \%$ of the HS associated with TB.
\end{abstract}

\section{Introduction}

Hemophagocytic syndrome (HS) is a rare disorder of the immune system with an estimated incidence of one per 800,000 people/year and one to ten per 1 million in the pediatric population in Italy, Sweden, and the USA. ${ }^{1}$ HS may be under-diagnosed owing to variable clinical presentations, diagnostic criteria and a low level

Correspondence: Maurizio Soresi, Division of Internal Medicine and Specialities (Di.Bi.M.I.S.), University-Hospital of Palermo, via del Vespro 141, 90127 Palermo, Italy.

Tel.: +39.91.6552961 - Fax: +39.91.6552896.

E-mail: maurizio.soresi@unipa.it

Key words: Hemophagocytic syndrome; hemophagocytic lymphohistiocytosis disseminated tuberculosis; Mycobacterium tuberculosis.

Acknowledgments: we thank Dr. Virendra Singh Editor-inChief of Lung India for the permission of publishing Table 2.

Conflict of interest: the authors declare no potential conflict of interest.

Received for publication: 4 October 2017

Accepted for publication: 15 December 2017.

This work is licensed under a Creative Commons Attribution NonCommercial 4.0 License (CC BY-NC 4.0).

C Copyright V. Lombardo et al., 2018

Licensee PAGEPress, Italy

Italian Journal of Medicine 2018; 12:61-66

doi:10.4081/itjm.2018.932 of awareness by medical personnel, thus delaying prompt treatment and contributing to the high mortality rates. ${ }^{2}$ The main causes differ in each country, and this suggests a specific genetic background or differences in the circulation of suspected triggering agents, particularly infections. ${ }^{1}$ It is caused by a disorder of the immunoregulatory capacity. It is characterized by persistent fever, lymphadenopathy, hepatosplenomegaly, cytopenia and hyperferritinemia. ${ }^{1}$ It can be associated with infections, malignancies or autoimmune diseases. Among the rare causes of this syndrome there is tuberculosis (TB), whose diagnosis becomes especially complicated when the clinical picture does not clearly involve lung. TB infection is still common and remains an important cause of morbidity and mortality worldwide and particularly in underdeveloped and developing nations. The gastrointestinal tract is the sixth commonest extrapulmonary site to be affected after lymphatic, genitourinary, bones and joints, miliary and meningeal involvement. Manifestations can be non-specific and mimic many conditions, including malignancies. ${ }^{3}$ We describe a case of HS associated with disseminated Mycobacterium tuberculosis (MT) infection.

\section{Case Report}

A 33-year-old Sudanese man, who had been living in Italy for 3 years, was admitted to a hospital in another Italian region for abdominal pain unresponsive after unspecified treatment, diarrhea and weight loss. Esophagogastroduodenoscopy and colonoscopy were negative, so he was discharged with no specific therapy. Because 
of the persistence of symptoms and a continuous intermittent fever $\left(39^{\circ} \mathrm{C}\right)$ onset 15 days before, he was admitted to our Department of Internal Medicine. His past medical history was unremarkable. He was ex-smoker and drinker; he did not use drugs. On admission, his clinical conditions were poor; he was anorexic. His weight was $50 \mathrm{~kg}$, his height was $180 \mathrm{~cm}$, body mass index 15.4. His blood temperature was $38^{\circ} \mathrm{C}$. Abdominal palpation revealed pain in the mesogastric region. Abnormal laboratory values were: hemoglobin 8.4 $\mathrm{g} / \mathrm{dL}$, white bood cells $1200 / \mathrm{mmc}$, platelets $130,000 / \mathrm{mmc}$, erythrocyte sedimentation rate 29 , ferritin $1700 \mathrm{ng} / \mathrm{mL}$, triglycerides $315 \mathrm{mg} / \mathrm{dL}$, reduced serum albumin, calcium and sodium, positive fecal occult blood test. An extensive serological screening was performed with the aim to identify any possible cause of persistent fever: multiple blood cultures (all negative), serologic tests for infectious diseases [cytomegalovirus (CMV), Epstein-Barr (EBV), hepatitis B and C, HIV viruses, Salmonella typhi, resulted all negative], negative was the MT research in the urine and in the blood and X-ray of the chest was negative too. Abdominal ultrasound showed: splenomegaly (spleen $13.5 \mathrm{~cm}$ ), numerous mesenteric lymph nodes enlargement (the maximum longitudinal diameter was 2-3 cm). Some small bowel loops were dilated and thickened. There was also a thin ascites and small bilateral pleural effusion. A computed tomographic scan of the abdomen confirmed this ultrasound picture. Meanwhile, a culture of pharyngeal gargle became positive for Pneumocystis carini, so we started antibiotic therapy with sulfamethoxazole/trimethoprim 1600 $\mathrm{mg}$ /die and ciprofloxacin $400 \mathrm{mg} / \mathrm{die}$ ev. The presence of a positivity to $P$. carini and the negativity of HIV serologic tests addressed us to formulate the hypothesis of lymphoma as a cause of the immunodepression state. In this suspicion a bone marrow biopsy was performed. Histological specimens of iliac crest showed signs of HS (Figures 1 and 2). So, we started therapy with prednisone $25 \mathrm{mg} /$ day with an initial improvement of blood cells values. Despite clinical improvement, some doubts arose: the syndrome is more often associated with infections than with lymphoma, ultrasound and computed tomography scans did not show the typical aspects of lymphoma and the presence of pleural involvement shifted the focus to the chest. At this point, our attention was focused on HS-associated infections and, after a review of the literature, despite the initial negative results of cultural tests, a MT infection was hypothesized as the trigger for HS. The bronchoalveolar lavage (BAL) culture that resulted positive for the first time for MT, was repeated again. The cultural test demonstration of the presence of MT, the radiological imaging compatible with TB have led us to the diagnosis of disseminated TB with predominant abdominal involvement associated with $H S$. The patient was treated with quadruple antitubercular therapy and ciprofloxacin, after 30 days the steroid was suspended and it was maintained the triple therapy. Signs of hemophagocytic syndrome improved. However, despite the quadruple therapy and the initial improvement after steroid therapy, patient's clinical conditions returned to be precarious and the imaging study showed a significant worsening; splenic lesions increased in volume and there was a worsening of the pulmonary imaging framework. The patient died before being able to perform a new BAL to assess the possible presence of MB resistant to conventional therapy.

\section{Discussion}

Hemophagocytic syndrome or hemophagocytic lymphohistiocytosis is an example of a failure of the immune response that leads to a severe condition of hyper-inflammation. The disease develops because the $\mathrm{T}$ cells proliferate in an aberrant way, causing the hyper-activation of macrophages which increase their phagocytic activity of blood cells (especially erythrocytes); hence the name of the syndrome. ${ }^{4}$

Depending on the etiology, HS can be divided into genetic (i.e., primary) and acquired (i.e., secondary) forms. Genetic and familial forms (primary) have an early onset, they recognize a genetic defect, and can be triggered by viral infections. The acquired forms (or secondary) can occur at any age, many times there is not an underlying immune deficiency. The acquired form is found in association with malignancies (especially T-cell lymphoma), autoimmune diseases, drug hypersensitivity reactions or infections. ${ }^{5}$ The trigger of hyper-inflammation can be represented not only by viral infections ( $43 \%$ of the viral cases are due to EBV and $9 \%$ to $\mathrm{CMV})^{1}$ but also by a variety of other viral, bacterial and parasitic pathogens, including S. typhi, Histoplasma capsulatum, Leishmania, Toxoplasma gondii, HIV and $M$. tuberculosis.

The pathophysiological bases of the syndrome are still not clear. However, many reports suggest that abnormalities in the immune regulatory system play a central role in HS. In particular, an imbalance in Th1-Th2 cells, especially an uncontrolled augmentation of Th1 response and subsequent activation of cytotoxic $\mathrm{T}$ cell and macrophages, could be the pathophysiological mechanism behind the syndrome. ${ }^{6}$ Secondary forms of HS are often triggered by intracellular pathogens that typically activate an immune response of type Th1. ${ }^{7}$ In animal models, immunological control of MT infection requires potent Th1 cell immunity. So, it is likely that the altered immune response, in patients with tuberculosis, may trigger the syndrome. ${ }^{8}$

In our report the hypothesis of TB was supported by: chest involvement, the co-existence of disseminated TB with HS, the high incidence in Sudan. 


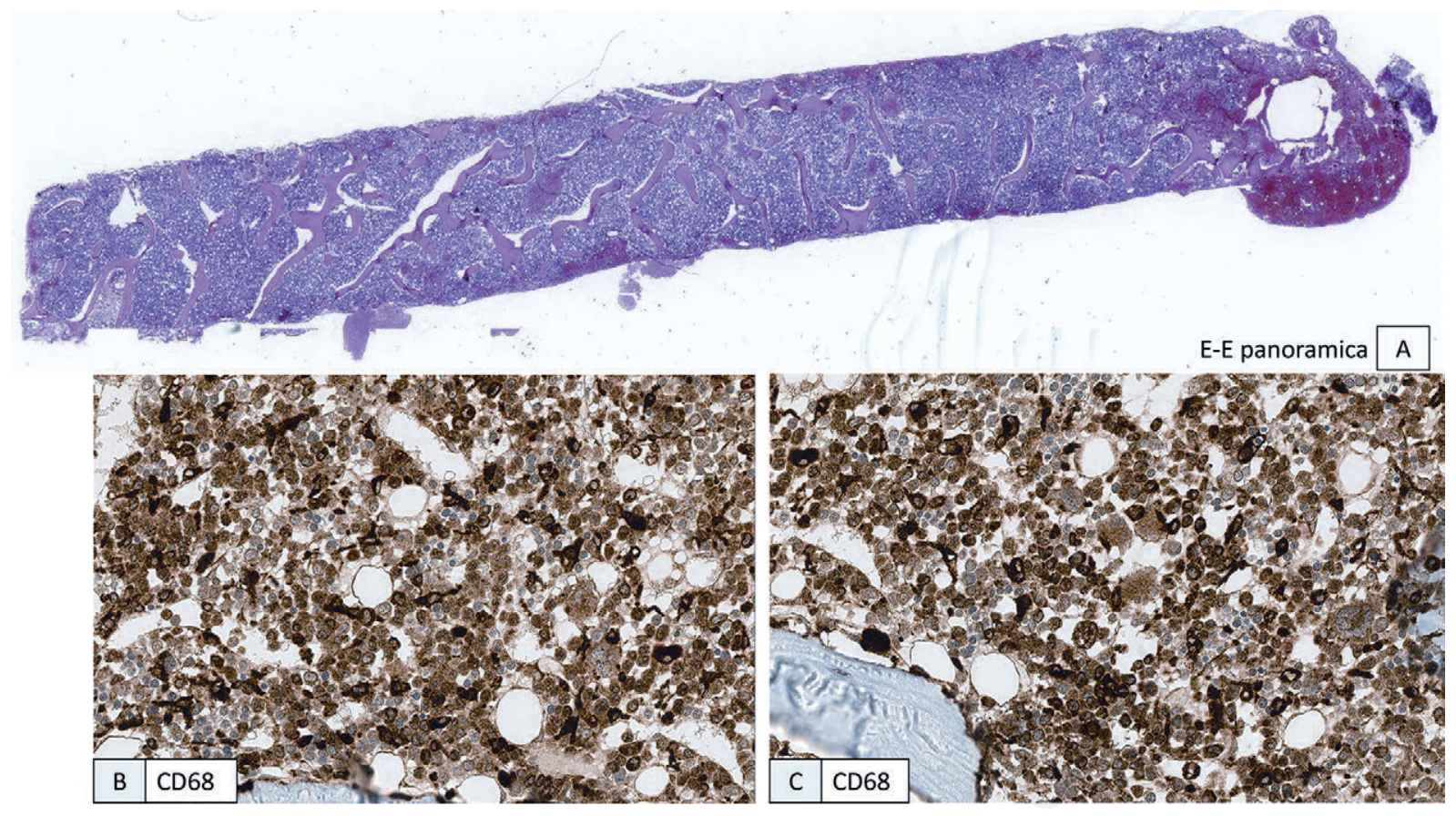

Figure 1. A) E-E overview: bone marrow biopsy showing increased cellularity, evaluated $80 \%$ (normal value: average $50 \%$ ). B and C) CD68: the pictures show several histiocytes (marked by anti-CD68 antibody) demonstrating phagocytosis of red blood cells and granulocytes.
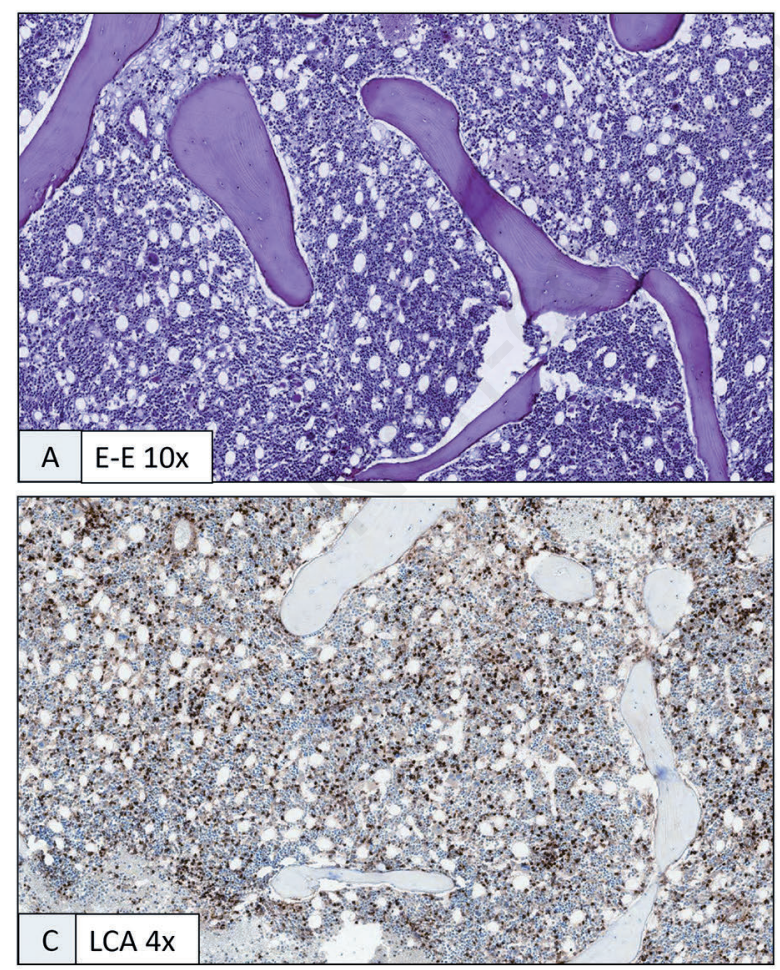
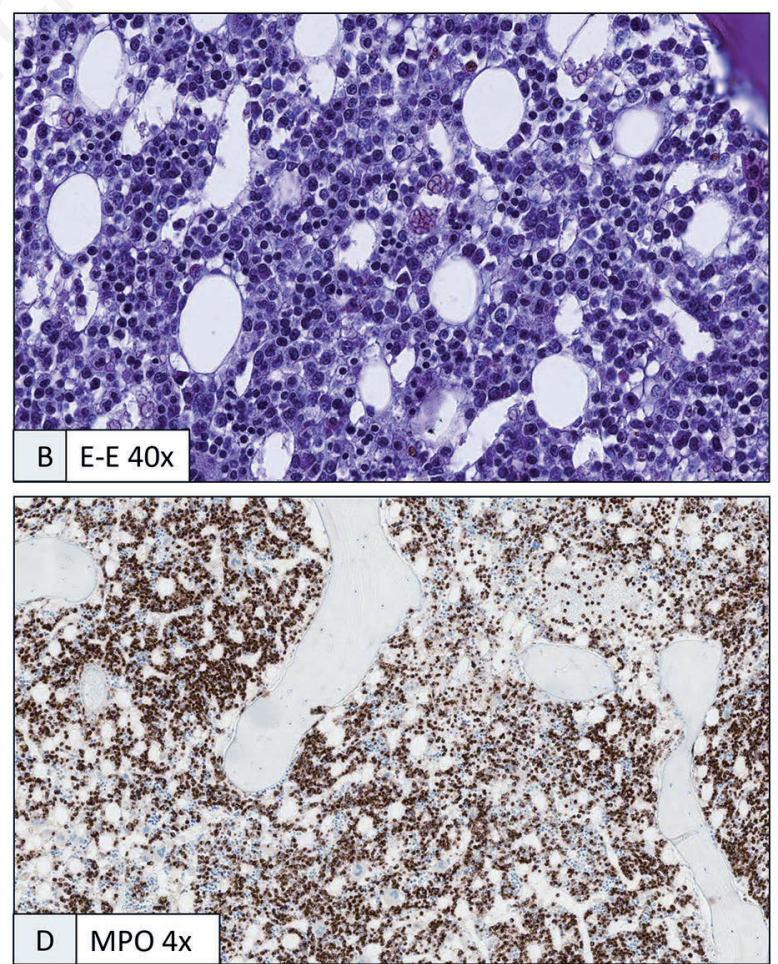

Figure 2. A) E-E 10×: detail of increased bone marrow cellularity with a normal distribution of megakaryocytes. B) EE 40×: higher magnification shows normal maturing bone marrow cellular elements (myeloid, erythroid and megakaryocytic components) associated with a pronounced histiocytic hyperplasia, focally demonstrating phagocytosis. C) LCA: staining with anti-CD45 antibody (LCA) shows a reactive lymphoid tissue with interstitial e micronodular distribution. D) MPO: bone marrow cellularity is largely composed by myeloid component (marked by anti-MPO antibody) which is more represented than the erythroid counterpart (myelo-erythroid ratio 4:1). 
The difficulty of making diagnosis in this case is related to several facts. First, the low prevalence of disseminated TB (which is estimated to be between 0.6 and $8 \%$ ), and even more in the clinical presentation of our patient: absence of pulmonary symptoms (lack of cough, clear pleural effusion only after a long time), the coexistence of an HS which complicated a severe underlying disease: severe pancytopenia, the difficulty in isolating the germ. Lastly, perhaps our approach to the formulation of Western-type diagnostic hypotheses has been misleading. Indeed, supposing an immunodepression (coinfection with P. carinii) the negativity of HIV and the presence of HS induced us to think about a lymphoma rather than a TB.

Diagnostic criteria of HS are the following: fever, splenomegaly, cytopenia (at least 2 lines), hypertriglyceridemia and/or hypofibrinogenemia, hemophagocytosis in bone marrow or spleen or lymph nodes. In addition, compared to the 5 criteria of 1991, three additional criteria have been introduced between 2004 and 2017 (Table 1). ${ }^{9,10}$

In our clinical case and laboratory, parameters satisfied 5 out of 8 diagnostic criteria of HS. In addition, even one of the new diagnostic criteria was satisfied. Highly elevated ferritin is strongly associated with HS and compared to the other two parameters [natural killer-cell activity and interleukin (IL)-2 receptor levels] is more available in everyday practice. ${ }^{11}$ In fact the goal is to develop diagnostic criteria able to identify the disease at an early stage before the storm of proinflammatory cytokines has developed. ${ }^{12}$

All symptoms can be explained by the high concentration of pro-inflammatory cytokines and tissue infiltration by lymphocytes and activated histiocytes. ${ }^{13}$ The cardinal symptoms are: fever (caused by the release of IL-1 and IL-6), cytopenia (tumor necrosis factor- $\alpha, \gamma$-interferon), hepatosplenomegaly (secondary to tissue infiltration). Other symptoms include: lymphadenopathy, rash, jaundice, neurological symptoms. Biochemical parameters include: high levels of triglycerides, ferritin, transaminases, bilirubin and lactate dehydrogenase, and low levels of fibrinogen. ${ }^{5}$ Fever and hepatosplenomegaly are present in $70 \%$ of patients at an early stage of the disease while cytopenia, hypertriglyceridemia and hyperferritinemia are present in 50\% and hemophagocytosis in about $40 \%$ of patients as initial signs. This makes it very difficult to diagnose the syndrome, because fever and hepatosplenomegaly are very generic signs, which are present in many diseases. Moreover, the same manifestations can be caused by the disorder that triggers HS. The differential diagnosis is with infections (EBV, CMV, Leishmaniasis), leukemia, anaplastic large cell lymphoma, juvenile rheumatoid arthritis.

A review of the world literature showed, from January 1975 to March 2014, 63 cases of TB associated

Table 1. Revised diagnostic guidelines for hemophagocytic lymphohistiocytosis. ${ }^{10}$

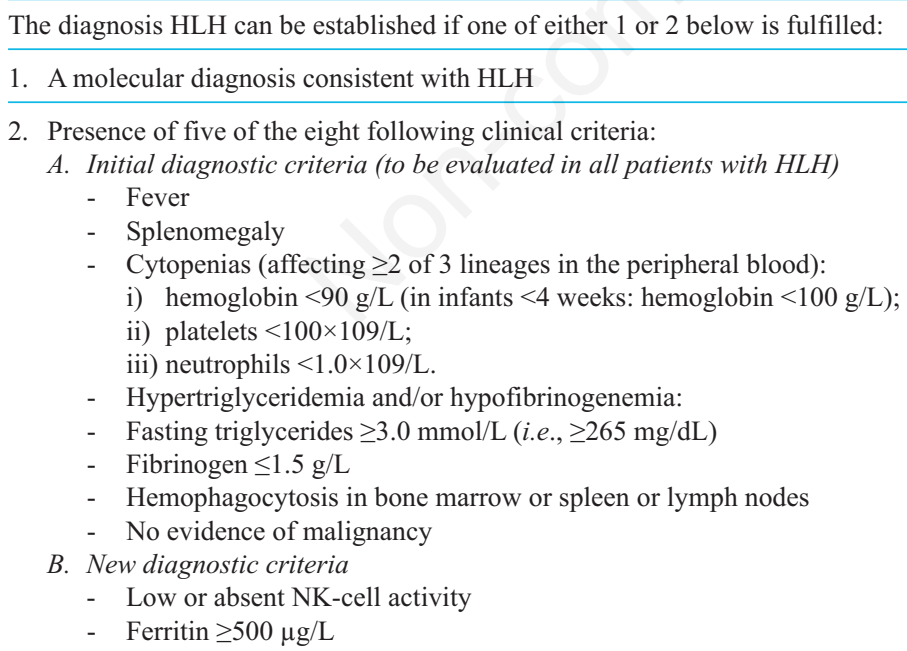

3. Soluble CD25 (i.e., soluble IL-2 receptor) $\geq 2400 \mathrm{U} / \mathrm{mL}$ :

A. FHL should have no evidence of malignancy

B. Supportive evidence

- CNS symptoms and spinal fluid pleocytosis (mononuclear cells) and/or elevated spinal fluid protein

- Jaundice:

i) hepatic enzyme abnormalities and histological picture resembling

ii) chronic persistent hepatitis (biopsy), coagulopathy, lymph node

iii) enlargement, edema, skin rash, hypoproteinemia, hyponatremia

C. Absence of hemophagocytosis does not exclude a diagnosis of $H L H$

HLH, hemophagocytic lymphohistiocytosis; NK, natural killer; FHL, familial hemophagocytic lymphohistiocytosis; CNS, central nervous system. 
Table 2. Clinicopathological characteristics of patients with tuberculosis-associated hemophagocytic lymphohistiocytosis (January 1975 - March 2014).

\begin{tabular}{ll}
\hline Parameters & Values \\
\hline Number cases & $\mathrm{N}=63$ \\
\hline Men age (range) & 55 years (14 days-80 years) \\
\hline Associated co-morbidity & $65 \%$ \\
\hline Mean duration of symptoms before diagnosis $\%$ (range) & 51 days (7-180 days) \\
\hline Fever & $100 \%$ \\
\hline Splenomegaly \pm Hepatomegaly & $71 \%$ \\
\hline Management & $26 \%$ only ATT \\
& $59 \%$ ATT + immunosuppressor/immunomodulator \\
& $12.9 \%$ no therapy \\
\hline Outcome & $0.16 \%$ only immunotherapy \\
\hline
\end{tabular}

Modified from Padhi et al., $2015^{14}$ with permission.

with HS (Table 2). ${ }^{14}$ The mean duration of symptoms before diagnosis of TB-HS was 51 days and in 8 patients the diagnosis of TB was made at autopsy. So, the rarity, the multiform clinical presentation and the lack of knowledge about this disease, delay the diagnosis and consequently the beginning of therapy. Outcome was death in $49 \%$ of patients and in most cases was attributed to a delay in the beginning of therapy in the course of the illness. ${ }^{14}$

There is no consensus about the optimal treatment. In every case, the underlying disease must be aggressively treated, to arrest the development of HS and TB should be considered early in the differential diagnosis of infectious etiologies possibly associated with HS. ${ }^{2,12}$

\section{Conclusions}

The current epidemiology of tuberculosis in Italy is characterized by a low incidence in the general population and the concentration of most of the new cases in certain risk and age groups. $52 \%$ of the total reported cases occurred in foreign patients. On average $40 \%$ of the cases notified in foreign patients happen in the first two years from the arrival in our country. Tuberculosis is associated with significant mortality and morbidity rates, which increase if complicated by HS. Recognizing TB as the cause of HS, that is not easy when there is no pulmonary involvement, is crucial to start early treatment and improve prognosis. This case leads us to conclude that the diagnosis of tuberculosis should always be considered in patients at risk even when there is not a clear pulmonary involvement. The HS in disseminated TB is severe and can lead to the patient's death, the evolution of the clinical picture, however, shows though as disseminated TB has a poor prognosis and how only an early diagnosis can provide to these patients a safe recovery.

\section{References}

1. Ramos-Casals M, Brito-Zerón P, López-Guillermo A, et al. Adult haemophagocytic syndrome. Lancet 2014;383: 1503-16.

2. Price B, Lines J, Lewis D, Holland N. Haemophagocytic lymphohistiocytosis: A fulminant syndrome associated with multiorgan failure and high mortality that frequently masquerades as sepsis and shock. S Afr Med J 2004;6:401-6.

3. Kapoor VK. Abdominal tuberculosis. Postgrad Med J 1998;74:459-67.

4. Certica V, Aricò M. Sindrome emofagocitica. Hematol Meeting Rep 2008;2:75.

5. Janka GE. Hemophagocytic syndromes. Blood Rev 2007;21:245-53.

6. Tsuda H, Fujisao S. Th1/Th2 mileu in adult hemophagocytic syndrome. Acta Haematol 1999;101:157-60.

7. Brastianos PK, Swanson JW, Torbenson M, et al. Tuberculosis-associated haemophagocytic syndrome. Lancet Infect Dis 2006;6:447-54.

8. Jung YJ, Ryan L, LaCourse R, North RJ. Properties and protective value of the secondary versus primary $\mathrm{T}$ helper type 1 response to airborne Mycobacterium tuberculosis infection in mice. J Exp Med 2005;201:1915-24.

9. Henter JI, Horne AC, Arico M, et al. HLH-2004: Diagnostic and therapeutic guidelines for hemophagocytic lymphohistiocytosis. Ped Blood Cancer 2007;48:124-31.

10. Ramachandran S, Zaidi F, Aggarwal A, Gera R. Recent advances in diagnostic and therapeutic guidelines for primary and secondary hemophagocytic lymphohistiocytosis. Blood Cells Mol Dis 2017;64:53-7.

11. Allen CE, Yu X, Kozinetz CA, et al. Highly elevated ferritin levels and the diagnosis of hemophagocytic lymphohistiocytosis. Pediatr Blood Cancer 2008;50: 1227-35. 
12. Larroche C. Hemophagocytic lymphohistiocytosis in adults: diagnosis and treatment. Joint Bone Spine 2012; 79:356-61.

13. Schneider EM, Lorenz I, Muller-Rosenberger M, et al. Hemophagocytic lymphohistiocytosis is associated with deficiencies of cellular cytolysis but normal expression of transcripts relevant to killer-cell-induced apoptosis. Blood 2002;100:2891-8.

14. Padhi S, Ravichandran K, Sahoo J, et al. Hemophagocytic lymphohistiocytosis: An unusual complication in disseminated Mycobacterium tuberculosis. Lung India 2015;32:593-601. 